\title{
Long Non-Coding RNA CCAT1 Acts as a Competing Endogenous RNA to Regulate Cell Growth and Differentiation in Acute Myeloid Leukemia
}

\author{
Lianxiang Chen ${ }^{1, *}$, Wei Wang', Lixia Cao', Zhijun Li ${ }^{\beta}$, and Xing Wang ${ }^{3}$
}

\begin{abstract}
Long non-coding RNAs (IncRNAs) are involved in multiple cellular events, as well as in tumorigenesis. Colon cancerassociated transcript-1 (CCAT1) gene encodes an IncRNA whose over-activation was observed in an expanding list of primary human solid tumors and tumor cell lines, however its biological roles in acute myeloid leukaemia (AML) has not been reported yet at present. In this study, the aberrant upregulation of CCAT1 was detected in FrenchAmerican-British M4 and M5 subtypes of adult AML patients. By gain- and loss-of-function analysis, we determined that CCAT1 repressed monocytic differentiation and promoted cell growth of HL-60 by sequestering tumor suppressive miR-155. Accordingly, a significant decrease in miR-155 level was detected in AML patients. Reintroduction of miR-155 into HL-60 cells restored monocytic maturation and repressed cell proliferation. Furthermore, CCAT1 could up-regulated c-Myc via its competing endogenous RNA (ceRNA) activity on miR-155. In conclusion, these results revealed new mechanism of IncRNA CCAT1 in AML development, and suggested that the manipulation of CCAT1 expression could serve as a potential strategy in AML therapy.
\end{abstract}

\section{INTRODUCTION}

Acute myeloid leukemia (AML) is a clonal neoplastic disorder that is characterized by increase in myeloid cell numbers in patients bone marrow and arrest in cell maturation, frequently leading to hematopoietic insufficiency (Saadatpour et al., 2014). It is likely that many different genomic mutations, epigenetic aberrations, or abnormalities in gene expression could contribute to AML progression. Previous studies have

\footnotetext{
'Department of Hematology, The Affiliated Hospital of Inner Mongolia Medical University, Hohhot 010059, China, 'Department of Emergency, Inner Mongolia People's Hospital, Hohhot 010017, China, ${ }^{3}$ Department of Anatomy, Basic Medical College, The Inner Mongolia Medical University, Hohhot 010059, China

*Correspondence: tubageww@ sina.com (LXC)
}

Received 9 November, 2015; revised 4 February, 2016; accepted 11 February, 2016; published online 26 February, 2016

Keywords: AML, CCAT1, IncRNA, miR-155 shown that the overgrowth of abnormal myeloid blasts and severe blockage in myeloid differentiation are the major hallmarks of AML (Ichikawa et al., 2004; Jeziskova et al., 2015; Sakurai et al., 2014; Su et al., 2015). Therefore, repressing cell growth or overcoming differentiation arrest by manipulating such key regulators becomes the major driving force of this blood cancer.

Recent studies on human transcriptome have demonstrated that a relevant part of the genome is transcribed into the heterogeneous class of long non-coding RNAs (IncRNAs). Similar to protein-coding genes, IncRNAs may have important roles in diverse cellular processes, with their dysregulation already associates with disorders, including leukemia (Khandelwal et al., 2015; Peng et al., 2015; Ye et al., 2014; Zhang et al., 2013). Thus, the investigation of expression and function of IncRNAs could help with our understanding of leukemogenesis and the identification of novel therapeutic targets. Until now, several IncRNAs have been identified in normal or malignant hematopoiesis (Fernando et al., 2015; Hughes et al., 2015; Wang et al., 2015). However, only a few have been mechanically and functionally characterized in the context of AML-associated aberrant gene networks (Hughes et al., 2015).

Colon cancer-associated transcript-1 (CCAT1), a 2kb IncRNA that maps to chromosome 8q24.21, was first found to be upregulated in colon cancer (Alaiyan et al., 2013; Yang et al., 2013). Recent study has suggested that CCAT1 was also upregulated in gastric and hepatocellular carcinoma tissues (Deng et al., 2015; Mizrahi et al., 2015; Zhu et al., 2015). Although CCAT1 has pivotal roles in different cancers, little is known about its involvement in AML, and the mechanism by which CCAT1 exerts its oncogenic activity remains undefined. In this study, we showed that IncRNA CCAT1 was increased in AML patients, especially the M4 and M5 subtypes (the myelomonocytic subtypes), compared to the normal controls. We further demonstrated that CCAT1 inhibited myeloid cell differentiation and promoted cell proliferation by operating as a competing endogenous RNA (ceRNA) for the miR-155 microRNA (miRNA). C-Myc was subsequently validated as a downstream target of CCAT1 ceRNA activity, and was important for CCAT1 to regulate AML progression, suggesting that CCAT1 regulates miR-155 activity by altering its targeting Taken together, these results suggest that CCAT1 could regulate leukemogenesis as a ceRNA and may serve as a potential target for AML therapies. 


\section{MATERIALS AND METHODS}

\section{Human samples}

The peripheral blood samples from AML patients and normal volunteers were obtained from The Affiliated Hospital of Inner Mongolia Medical University. The informed consent to perform the biological studies was obtained from all of the examined subjects and the related study was approved by the Ethics Committees of the participating hospitals and the Institutional review Board of The Affiliated Hospital of Inner Mongolia Medical University.

\section{Cell lines and cell culture}

Human myeloid leukemia cell lines HL-60 was purchased from cell resource center of Shanghai Institutes for Biological Science. HL-60 was cultured in IMDM (Gibco, BRL, UK) supplemented with $10 \%$ FCS (Gibco), $50 \mathrm{U} / \mathrm{ml}$ penicillin and $50 \mu \mathrm{g} / \mathrm{ml}$ streptomycin (Sigma-Aldrich, USA) at $37^{\circ} \mathrm{C}$ in $5 \% \mathrm{CO}$. The monocytic differentiation of $\mathrm{HL}-60$ was induced by $50 \mathrm{ng} / \mathrm{ml}$ phorbol myristate acetate (PMA; Sigma-Aldrich).

\section{RNA isolation, reverse transcription and quantitative real-time PCR}

Total RNA was isolated from the cell harvest using Trizol agents (Invitrogen, USA) according to the manufacturer's instruction. Quantitative real-time PCR assay was conducted to detect the level of RNA transcripts. Briefly, cDNA was synthesized by M-MLV reverse transcriptase (Invitrogen) from 2 ug of total RNA. Oligo (dT18) RT primer was used for the reverse transcription of mRNA and IncRNA. Stem-loop RT primer was used for the reverse transcription of miR-155. Quantitative realtime PCR was carried out in the Bio-Rad IQ5 real-time PCR system (Bio-rad, USA) in triplicate with the following cycling conditions: $95^{\circ} \mathrm{C}$ for $10 \mathrm{~min}$ (initial denature); then 40 cycles of $95^{\circ} \mathrm{C}$ for $15 \mathrm{~s}, 60^{\circ} \mathrm{C}$ for $60 \mathrm{~s}$. The miR-155 specific forward primer sequence was designed on the basis of miRNA sequences obtained from the miRBase database. The data were normalized using the endogenous GAPDH or U6 snRNA for mRNA/IncRNA or miRNA, respectively.

\section{Plasmid constructs, Oligonucleotides and cell transfection} For gain-of-function analysis of CCAT1, the full length of CCAT1 was amplified from human cDNA and inserted into pcDNA6.1 (Invitrogen, USA) vectors. For loss-of-function analysis of CCAT1, shRNAs specific to CCAT1 was synthesized and inserted into pSIH (System Biosciences, USA) vectors. For miR-155 target analysis, the full length of CCAT1 was amplified and inserted into pMIR-REPORT vectors. The mutant CCAT1 constructs were generated by using the QuikChange site-directed mutagenesis kit (Stratagene, USA). miR-155 mimic and mimic control were purchased from GenePharma (China) and used at a final concentration of $25 \mathrm{nmol} / \mathrm{L}$ in transfection by using DharmaFECT 1 (Dharmacon; USA) in accordance with the manufacturer's instructions. HL-60 cells were also transfected with different CCAT1 constructs at a final concentration of $2 \mu \mathrm{g} / \mathrm{ml}$ using Lipo2000 (Invitrogen; USA) in accordance with the manufacturer's instructions. For the "rescue" assay, the pcDNA-CCAT1 constructs or empty vectors (final concentration of $2 \mu \mathrm{g} / \mathrm{ml}$ ) was co-transfected with miR-155 mimic or mimic control (final concentration of $10 \mathrm{nmol} / \mathrm{L}$ ) using Lipo2000 into HL-60 cells.

\section{Luciferase reporter assay}

HL-60 cells were co-transfected with pMIR constructs containing the wild type CCAT1 or diverse mutant CCAT1 (final concen- tration of $1 \mu \mathrm{g} / \mathrm{ml}$ ), along with the pRL-TK control vector (final concentration of $0.2 \mu \mathrm{g} / \mathrm{ml}$ ) and miR-155 mimic or mimic control. The plasmid pRL-TK containing Renilla luciferase was used as an internal control. Cells were harvested $48 \mathrm{~h}$ post-transfection and assayed with Dual Luciferase Assay (Promega, USA) according to the manufacturer's instructions. Data were obtained by normalization of renilla luciferase activity to firefly luciferase activity. All transfection assays were performed in triplicate.

\section{RNA pull-down by MS2-MBP}

Maltose-binding protein (MBP)-affinity purification was used in this study to identify miRNAs that associated with CCAT1. The MS2-MBP protein was expressed and purified from E. coli following a protocol from the Steitz lab. Three bacteriophage MS2 coat protein-binding sites (5'-cgtacaccatcagggtacgagctagcccatggcgtacaccatcagggtacgactagtagatctcgtacaccatcagggtacg-3') were inserted downstream of the wild type or mutant CCAT1 by site-directed mutagenesis using Stratagene's QuikChange SiteDirected Mutagenesis Kit. To obtain miRNAs associated with the MS2-tagged CCAT1, HL-60 cells were transfected with MS2-tagged CCAT1 constructs, and 10 million cells were used for each immunoprecipitation assay. The cells were harvested $48 \mathrm{~h}$ post-transfection and subjected to RNA pull-down analysis as described elsewhere (Peng et al., 2015).

\section{Western blot}

Total proteins were extracted from cells or tissues using RIPA buffer (50 mM Tris-HCl, pH 7.4, $150 \mathrm{mM} \mathrm{NaCl}, 1 \mathrm{mM}$ EDTA, $1 \%$ Triton $X-100,1 \%$ sodium deoxycholate, $0.1 \%$ SDS) supplemented with $1 \mathrm{mM}$ PMSF, $5 \mu \mathrm{g} / \mathrm{ml}$ aprotinin, $5 \mu \mathrm{g} / \mathrm{ml}$ leupeptin. The protein concentration was determined by BCA Protein Assay Kit (Vigorous, China). $20 \mu \mathrm{g}$ total protein was loaded to $10 \%$ SDS-PAGE gel, probed with mouse or rabbit mAb against c-Myc (Proteintech, 10828-1-AP), and GAPDH (Proteintech, 10494-1-AP) followed by horseradish peroxidase-(HRP) conjugated sheep anti-mouse or rabbit Ig (ZSGB-BIO).

\section{Flow cytometry analysis}

The harvested cells were washed with PBS, resuspended in PBS containing 1\% FCS and stained with CD11b-FITC and CD14-PE (Biogend, USA) for $30 \mathrm{~min}$ at $4^{\circ} \mathrm{C}$. Stained cells were sorted for expression of CD11b and CD14 using BD Accuri C6 flow cytometer. All the flow cytometry assays were carried out in triplicate.

\section{Cell proliferation assay}

HL-60 cells were incubated in 10\% CCK-8 (DOJINDO, Japan) diluted in culture medium at $37^{\circ} \mathrm{C}$ until visual color conversion occurred. Proliferation rates were determined at $0,24,48,72$, $96 \mathrm{~h}$ after transfection. The absorbance of each well was measured with a microplate reader set at $450 \mathrm{nM}$ and $630 \mathrm{nM}$. All experiments were performed in quadruplicate.

\section{Statistics}

Student's t-test (two-tailed) was performed to analyze the data. A two-sided $\mathrm{P}$-value of less than 0.05 was considered statistically significant. All statistical computations were performed using SPSS (SPSS Inc., USA).

\section{RESULTS}

CCAT1 was significantly increased in AML M4 and M5 subtype samples

To examine whether the CCAT1 was abnormally expressed in 

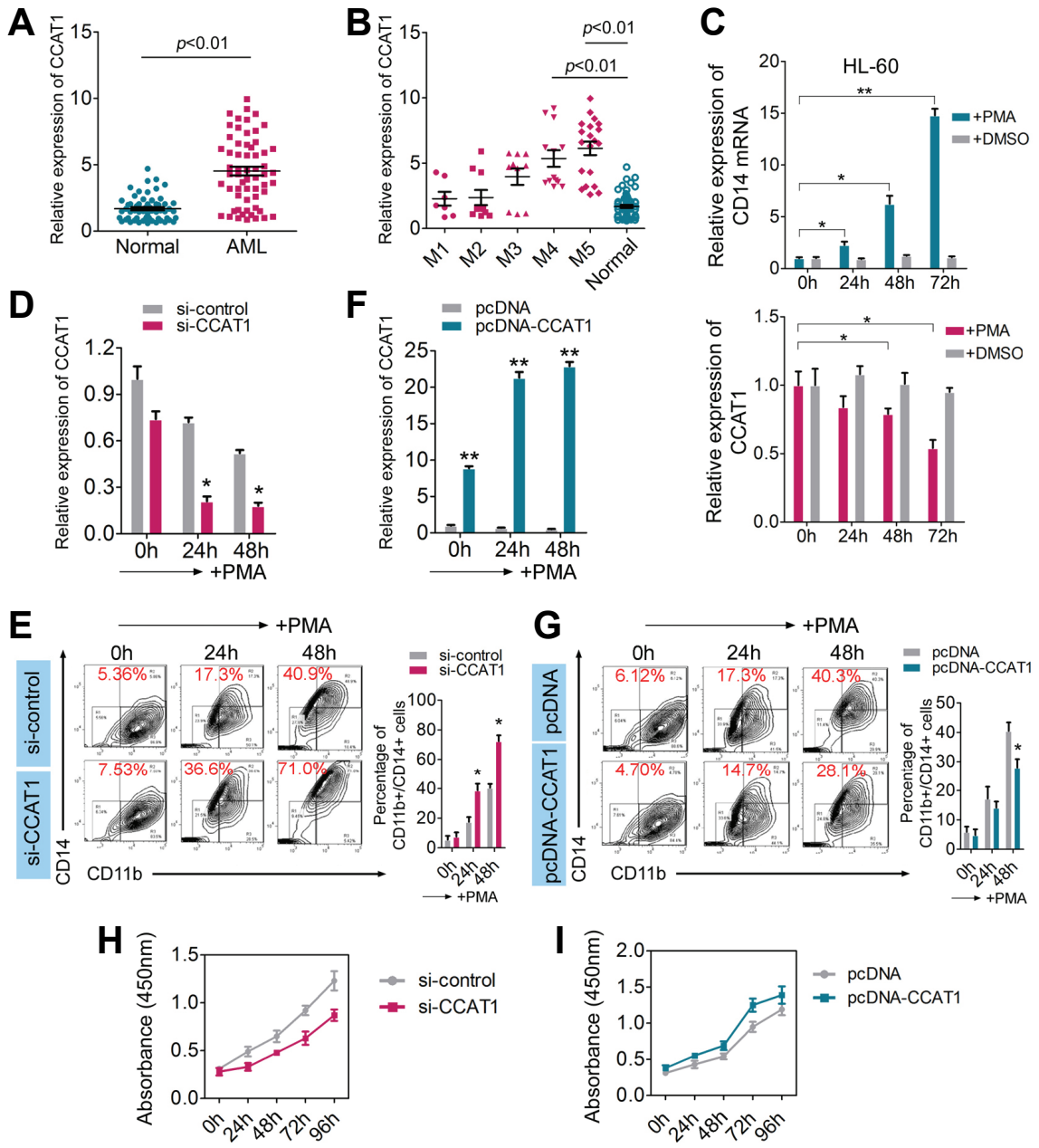

Fig. 1. Expression of CCAT1 in PBMNCs derived from AML patients (from FAB M1 to M5 subtypes) and healthy donors. (A) The expression levels of CCAT1 in PBMNCs derived from 60 AML patients and 60 healthy donors. Horizontal lines represented the means \pm s.d., and each dot represented an individual sample. (B) Expression of CCAT1 in M1, M2, M3, M4 and M5 AML samples were compared with that in the health controls, respectively. The expression data of CCAT1 were classified according to FAB subtypes. (C) The expression levels of CD14 (the upper panel) and CCAT1 (the lower panel) were analyzed by real-time PCR in HL-60 cells induced by PMA and its vehicle (dimethyl sulfoxide). (D) The expression levels of CCAT1 in HL-60 cells transfected with CCAT1 shRNAs (si-CCAT1) and control (si-control). (E) Flow cytometry analysis of CD11b and CD14 expression in HL-60 cells transfected with CCAT1 shRNAs and control. (F) The expression levels of CCAT1 in HL-60 cells transfected with a construct expressing CCAT1 (pcDNA-CCAT1) and control (pcDNA). (G) Flow cytometry analysis of CD11b and CD14 expression in HL-60 cells transfected with pcDNA-CCAT1 and pcDNA. (H) Cell proliferation assay of $\mathrm{HL}-60$ cells after treatment with si-CCAT1 or si-control by using CCK-8. (I) Cell proliferation assay of $\mathrm{HL}-60$ cells after treatment with pcDNA-CCAT1 or pCDNA by using CCK-8. For all quantitative results, the data are presented as the mean \pm SEM, and the error bars represent the standard deviation obtained from three independent experiments. ${ }^{*} p<0.05$; ${ }^{* *} p<0.01$.
AML, we performed real-time PCR to detect its level in peripheral blood mononucleic cells (PBMNCs) derived from 60 patients with newly diagnosed $A M L$ and 60 healthy donors. As shown in Figure 1A, CCAT1 was markedly upregulated in AML patients compared with the normal controls. These AML specimens represent the major French-American-British (FAB) subtypes from M1 to M5: 7 M1, 10 M2, 10 M3, 13 M4 and 20 M5. Furthermore, we compared CCAT1 level in different AML subtypes and found significantly increased CCAT1 expression mainly in the samples of M4 and M5 subtypes (Fig. 1B). The dysregulation of CCAT1 in AML M4 and M5 subtypes might suggest its potential oncogenic role in the myelomonocytic leukemogenesis.

Given that the AML M4 and M5 subtypes might involve monocyte-like differentiation arrest, we detected the expression changes of CCAT1 during monocytic differentiation of HL-60 leukemia cell lines. Real-time PCR data showed that CCAT1 level gradually decreased (Fig. 1C, the lower panel) during phorbol myristate acetate (PMA)-induced monocytic differentiation of HL-60 cells (Fig. 1C, the upper panel). To further test whether CCAT1 plays an important role in monocytic differentiation, shRNA specific to CCAT1 (si-CCAT1) was transfected into HL-60 cells and the efficiency of CCAT1 knockdown was subsequently confirmed by real-time PCR analysis (Fig. 1D). Flow cytometry data showed that CCAT1 knockdown resulted in higher expression of both CD11b and CD14 in $24 \mathrm{~h}$ and $48 \mathrm{~h}$ PMA-treated HL-60 cells, when compared with the control (Fig. 1E). On the contrary, the overpresence of CCAT1 (Fig. 1F) reduced the percentage of $C D 11 b+/ C D 14+H L-60$ cells after PMA treatment (Fig. 1G).

Next, we detected the influence of CCAT1 on leukemia cell proliferation by using CCK-8 assay. As a result, the repression of CCAT1 led to significant decrease in cell proliferation (Fig. $1 \mathrm{H})$, whereas the ectopic expression of CCAT1 increased HL60 cell growth (Fig. 1I).

\section{CCAT1 is physically associated with miR-155 in leukemia cells}

The ceRNA hypothesis posits that specific IncRNA can function as sinks for pools of active miRNAs, functionally liberating mRNA transcripts targeted by certain miRNAs (Arancio et al., 2014; Sen et al., 2014; Song et al., 2014). A recent study has indicated that CCAT1 could promote hepatocellular carcinoma progression by functioning as a let-7 ceRNA (Deng et al., 2015). 
A

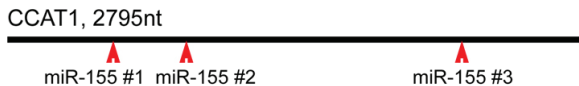

\begin{tabular}{|c|c|}
\hline Position & Base pairing \\
\hline $\begin{array}{c}\text { miR-155 \#1: } \\
205 \sim 211 \mathrm{nt}\end{array}$ & $\begin{array}{l}\text { CCAT1: 5' CAGCUGCCGUGUUAAGCAUUGCGAAAACGC 3' } \\
\text { I I I I I I I } \\
\text { miR-155: 3' UGGGGAUAGUGCUAAUCGUAAUU 5' }\end{array}$ \\
\hline $\begin{array}{c}\mathrm{miR}-155 \text { \#2: } \\
392 \sim 397 \mathrm{nt}\end{array}$ & $\begin{array}{l}\text { CCAT1: 5' UGCAAUCCAUCUGGAGCAUUCACUGACAAC 3' } \\
\text { I I I I I I I } \\
\text { miR-155: 3' UGGGGAUAGUGCUAAUCGUAAUU 5' }\end{array}$ \\
\hline $\begin{array}{l}\text { miR-155 \#3: } \\
\text { 1851 1859nt }\end{array}$ & $\begin{array}{l}\text { CCAT1: 5' GUCAGAUCAGCAGUGGCAUUAAAUUCUCAUA 3' } \\
\text { I I I I I I I I I I } \\
\text { miR-155: 3' UGGGGAUAGUGCUAAUCGUAAUU 5' }\end{array}$ \\
\hline
\end{tabular}

wild type: AGCAUU; mutant: CAGCAA

B

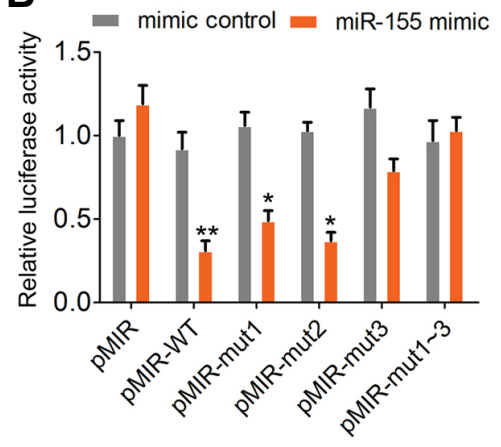

D
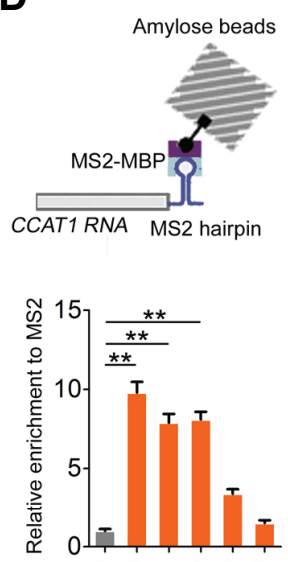

C

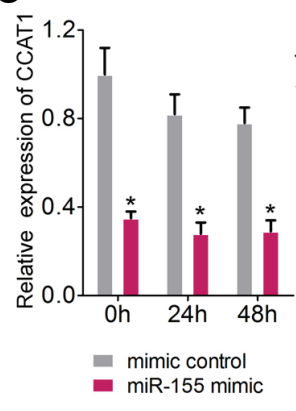

Fig. 2. The interaction of CCAT1 with miR-155. (A) The upper panel: schematic outlining the predicted binding sites of miR-155 on CCAT1. The lower panel: the presentation of miR-155's binding sites on CCAT1 transcript. The complementary sequences between CCAT1 and miR-155 were shown in bold. (B) Luciferase activity in HL-60 cells cotransfected with miR-155 mimic (25 nM) or mimic control (25 nM) and luciferase reporters containing nothing (pMIR), wild type CCAT1 (pMIR-WT) or diverse mutant CCAT1 as indicated. Data are presented as the relative ratio of firefly luciferase activity to renilla luciferase activity. (C) The expression levels of CCAT1 in HL-60 cells transfected with miR-155 mimic and mimic control. (D) MS2 RNA pull-down followed by real-time PCR to detect endogenous miR-155 associated with MS2-tagged CCAT1 transcript. For all quantitative results, the data are presented as the mean \pm SEM, and the error bars represent the standard deviation obtained from three independent experiments. ${ }^{*} p<0.05 ;{ }^{* \star} p<$ 0.01 .
To determine whether CCAT1 also operates as a ceRNA in leukemogenesis, we used the webserver InCeDB (http:// gyanxet-beta.com/lncedb/) to predict potential IncRNA-miRNA interactions. Among the results, we found 3 miR-155 binding sites scattering the CCAT1 transcripts, suggesting its ceRNA potential for another miRNA miR-155, in addition to let-7 (Fig. 2A). Subsequently, we constructed a series of luciferase reporters containing the wild type CCAT1 (pMIR -WT), or a series of mutant CCAT1 with mutations of a single (pMIR- Mut1, 2, 3) or all 3 predicted miR-155 binding sites (pMIR- Mut1 3). We found that transfection of miR-155 mimic into HL-60 cells reduced the luciferase activity of the wild type CCAT1 reporter (pMIR-WT) but not empty vector or all miR-155 site mutant reporter vector (Fig. 2B), suggesting the binding of miR-155 to these sites. Among them, the third miR-155 binding site (\#3) seemed to be the strongest interaction site, because the mutation of this sequence almost abolished the effects of miR-155 on luciferase activity (Fig. 2B). We next examined whether the endogenous CCAT1 level was affected by miR-155. As shown in Fig. 2C, CCAT1 expression was reduced by $\sim 3$-folds upon miR-155 over expression both before and after PMA induction (Fig. 2C). These results suggested that CCAT1 was negatively regulated by miR-155 in AML cells.

To further validate the direct interaction between miR-155 and CCAT1 at endogenous level, we performed an RNA pull down analysis to examine endogenous miRNAs associated with the MS2 sequence-tagged CCAT1. The precipitated miRNAs were analyzed by real-time PCR and results showed that miR-155 was significantly enriched by the MS2-tagged wildtype CCAT1 (pcDNA-WT-MS2) compared to the empty vector (pcDNA-MS2) or the all miR-155 targeting sites mutant ones
(pcDNA-Mut1 3-MS2) in HL-60 cells. These results suggested that endogenous miR-155 was associated with CCAT1 in leukemia cells (Fig. 2D). Accordingly, mutation of the third miR-155 binding site on CCAT1 resulted in a huge decrease in miRNA binding (Fig. 2D). Taken together, these data demonstrated that miR-155 could bind to CCAT1 and CCAT1 could function as a ceRNA in leukemia cells.

\section{miR-155 is downregulated in AML and functions as a tumor suppressor}

Based on above findings, miR-155 may also play an important role in leukemogenesis. We first assessed the expression changes of miR-155 in the same samples of $60 \mathrm{AML}$ patients and 60 healthy donors. As expected, miR-155 was significantly decreased in AML compared to normal controls (Fig. 3A). Interestingly, similarly to CCAT1, the lower miR-155 was specifically associated with M4 and M5 AML subtypes (Fig. 3B). Accordingly, miR-155 level was upregulated in PMA-induced monocytic differentiation of $\mathrm{HL}-60$ cells (Fig. $3 \mathrm{C}$ ), which was opposite to its ceRNA CCAT1.

Next, we still used HL-60 cells to analyze the role of miR-155 in monocytic differentiation. Real-time PCR was first used to measure the level of miR-155 after miR-155 mimic transfection and showed that the miR-155 increased to 40 to 60 -fold (Fig. 3D). Flow cytometry analysis showed that overexpression of miR-155 promoted monocytic maturation as indicated by the increased differentiated CD11b+/CD14+ cells (Fig. 3E). Furthermore, the CCK-8 proliferation assay in the same cells indicated that cell growth was suppressed after transfection with miR-155 mimic (Fig. 3F). Taken together, these results indicated the tumor suppressor role of miR-155 in AML. 

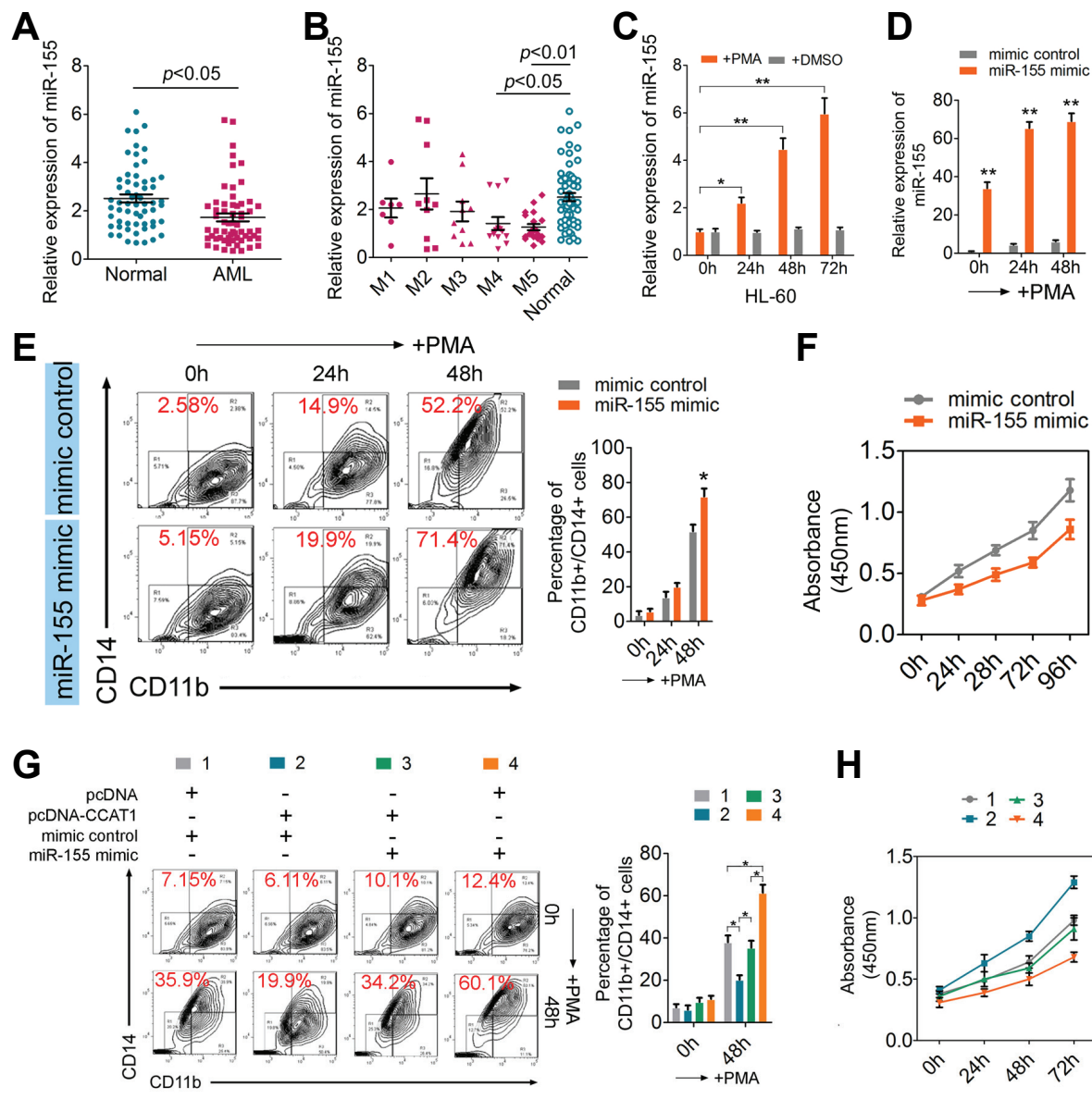

H

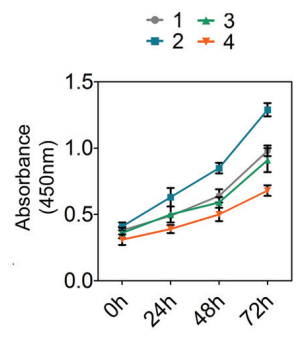

Fig. 3. Functional analysis of miR-155 in AML. (A) The expression levels of miR-155 in PBMNCs derived from 60 AML patients and 60 healthy donors. Horizontal lines represented the means \pm s.d., and each dot represented an individual sample. (B) Expression of miR-155 in M1, M2, M3, M4 and M5 AML samples were compared with that in the health controls, respectively. The expression data of miR-155 were classified according to $\mathrm{FAB}$ subtypes. (C) The expression level of miR-155 was analyzed by real-time PCR in HL60 cells induced by PMA and its vehicle (dimethyl sulfoxide). (D) The expression levels of miR-155 in HL-60 cells transfected with miR-155 mimic and control (mimic control). (E) Flow cytometry analysis of CD11b and CD14 expression in $\mathrm{HL}-60$ cells transfected with miR155 mimic and control. (F) Cell proliferation assay of $\mathrm{HL}-60$ cells after treatment with miR-155 mimic or control by using CCK-8. For all quantitative results, the data are presented as the mean \pm SEM, and the error bars represent the standard deviation obtained from three independent experiments. ${ }^{*} p<0.05$; ${ }^{*} p<$ 0.01. (G) Flow cytometry analysis of CD11b and CD14 expression in "rescue" assay as indicated. $(\mathrm{H})$ Cell proliferation assay of $\mathrm{HL}-60$ cells in "rescue" assay as indicated.
Furthermore, to detect whether the functional relevance of miR-155 in AML is regulated by CCAT1's ceRNA activity, we performed a "rescue" assay with co-transfection of pcDNACCAT1 and miR-155 mimic into HL-60 cells, followed by PMA treatment. As expected, the introduction of CCAT1 could rescue the miR-155-induced monocytic maturation of HL-60 cells (Fig. 3G), as well as the repression on cell proliferation driven by miR-155 (Fig. 3H). These results suggest that the differentiation-promoting and cell growth-repressing effects of miR-155 is closely connected to CCAT1 regulation in leukemia cells.

\section{CCAT1 ceRNA activity regulates c-Myc expression in \\ leukemia cells}

Studies have reported that miR-155 could control c-Myc expression in gastric carcinoma cells (Sun et al., 2014). To validate whether miR-155 also targets c-Myc in AML cells, realtime PCR and immunoblotting assay was carried out respectively, and showed that both the mRNA and protein levels of cMyc were decreased by 2-fold in HL-60 cells transfected with miR-155 mimic compared with the control (Fig. 4A). As a ceRNA, CCAT1 could share the regulatory miR-155 with its target c-Myc, therefore, we wondered whether CCAT1 could influence c-Myc in HL-60 cells. As excepted, overexpression of CCAT1 (pcDNA-CCAT1) increased both the mRNA (Fig. 4B) and protein levels of c-Myc (Fig. 4C). Furthermore, ectopic expression of miR-155 upon pCDNA-CCAT1 transfection abrogated this increase (Figs. $4 \mathrm{~B}$ and $4 \mathrm{C}$ ). All these results suggest that the role of CCAT1 in modulating c-Myc is mediated by its competitively binding with miR-155 in HL-60 cells.

Since the oncogenic role of CCAT1 was, at least partly, mediated by its ceRNA activity on miR-155/c-Myc axis, we supposed that the upregulated CCAT1 expression in AML would ultimately led to c-Myc accumulation. To test this hypothesis, we detected the level of c-Myc mRNA in AML samples. In consistence with CCAT1, c-Myc was markedly increased in AML patients compared to the normal control (Fig. 4D). However, only the AML M5 subtype had significantly higher level of c-Myc mRNA (Fig. 4E). In summary, these data indicated that CCAT1 inhibited myeloid cell differentiation and promoted cell proliferation by operating as a ceRNA for the miR-155, thus altering its target c-Myc.

\section{DISCUSSION}

The last decade has seen exciting advance in IncRNA research, suggesting that the involvement of IncRNA in human diseases could be far more prevalent than previously appreciated (Zhang et al., 2013). Different studies have shown that changes in IncRNA expression may contribute to the development of different types of cancer, however most of them have not yet been studied in mechanistic details. Recently, it has been demonstrated that endogenous IncRNA could participate in post-transcriptional regulation by interfering with the miRNA pathways, by acting as competing endogenous RNAs (ceR- 


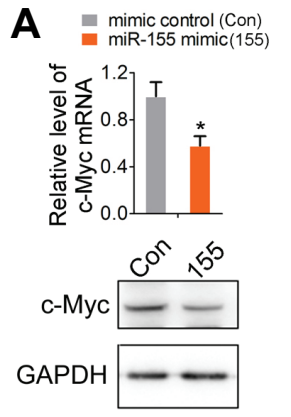

B

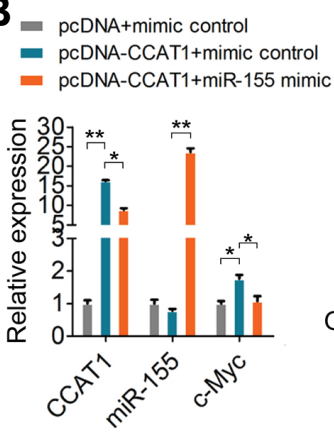

C

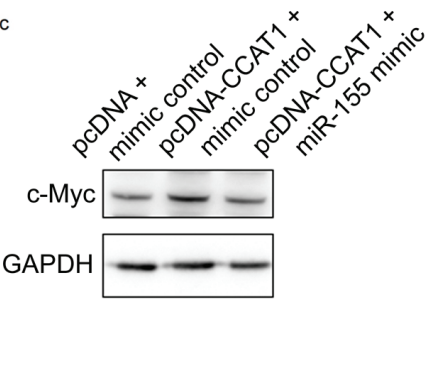

D

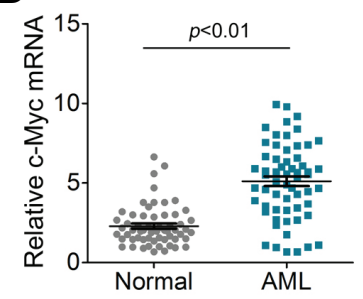

E

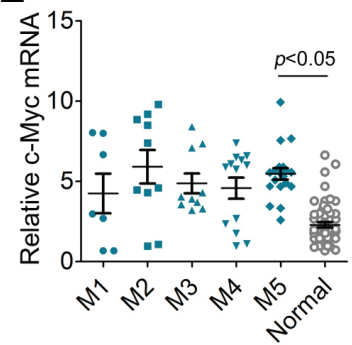

Fig. 4. Regulation of c-Myc by CCAT1. (A) The upper panel: The expression level of c-Myc mRNA was analyzed by real-time PCR in HL-60 cells transfected with miR-155 mimic or mimic control; The lower panel: Immunoblot analysis of cMyc ptotein in HL-60 cells transfected with miR-155 mimic or mimic control; An unrelated protein GAPDH was used as the control. (B) The expression of CCAT1, miR-155 and c-Myc mRNA in HL-60 cells transfected with control, wild type CCAT1 or wild type CCAT1 plus miR-155 mimic as indicated; (C) Immunoblot analysis of c-Myc protein in HL-60 cells treated as described in (B); (D) The expression levels of c-Myc mRNA in PBMNCs derived from $60 \mathrm{AML}$ patients and 60 healthy donors. Horizontal lines represented the means \pm s.d., and each dot represented an individual sample. (B) Expression of c-Myc mRNA in M1, M2, M3, M4 and M5 AML samples were compared with that in the health controls,

Myc were classified according to FAB subtypes. For all quantitative results, the data are presented as the mean \pm SEM, and the error bars represent the standard deviation obtained from three independent experiments. ${ }^{*} p<0.05$; ${ }^{* \star} p<0.01$.

NAs)(Arancio et al., 2014; Sen et al., 2014). These IncRNAs have miRNA responsive elements (MRE) and act as miRNA sponges to modulate miRNAs available for binding with their own targets (Johnsson et al., 2013). It is also suggested that these ceRNAs are implicated in many biological processes and the disruption of the equilibrium of ceRNAs and miRNAs is critical for tumorigenesis (Johnsson et al., 2013; Yokoyama et al., 2000). For example, IncRNA PTEN-P1 could act as ceRNA of miR-19b and miR-20a to release PTEN tumor suppressor in prostate cancer, glioblastoma, and melanoma, and disruption in the network leads to tumorigenesis in many cases (Poliseno et al., 2010; Yu et al., 2014). HULC IncRNA also blocked miR-372 from binding to its target PRKACB that induces the activation of CREB to modulate self-regulation in hepatocellular carcinoma (Wang et al., 2010). Similar report suggested that $\mathrm{H} 19$ acted as a ceRNA to modulate let-7 availability (Kallen et al., 2013). In a recent study, IncRNA-ATB upregulated ZEB1 and ZEB2 by competitively binding to the miR-200 family and then induced EMT and invasion (Yuan et al., 2014). Additionally, Hmga2 promotes lung carcinogenesis both as a protein-coding gene and as a ceRNA dependent upon the presence of let-7 sites (Kumar et al., 2014). Thus, understanding the intricate interplay among diverse RNA species, including protein-coding messenger RNAs and non-coding RNAs such as long non-coding RNAs, pseudogenes and miRNAs will lead to significant insight into gene regulatory networks and have implications in human disease.

CCAT1 is an IncRNA initially characterized in colon cancer. Recently, CCAT1 was found to be crucial for cancer cell invasion and proliferation in both gastric and hepatocellular carcinoma (Mizrahi et al., 2015; Yang et al., 2013; Zhu et al., 2015). However, little is known about the involvement of CCAT1 in leukemia, and the mechanism through which CCAT1 exerts its oncogenic activity remains undefined. Here, we identified CCAT1 as a novel regulator of acute myeloid leukemogenesis.
More importantly, we demonstrated a ceRNA activity of CCAT1 by modulating miR-155 availability in leukemia cells. Furthermore, the oncogenic role of CCAT1 was reinforced by upregulating the expression of c-Myc, a target of miR-155. Interestingly, CCAT1 has been reported to be activated by c-Myc in gastric carcinoma, suggesting the existence of a feedback loop consisted of c-Myc, CCAT1, miR-155, which is likely to be validated in future studies.

Another important finding in this study is to provide novel evidence for a tumor suppressive role of miR-155 in AML by inhibiting cell proliferation and releasing the monocytic differentiation block. miR-155 has been reported to be an oncogenic miRNA due to its up-regulation in diffuse large $B$ cell lymphoma (Eis et al., 2005), Hodgkin lymphoma (Kluiver et al., 2005), CLL (Calin et al., 2005), and AML with FLT3-ITD mutations (Gerloff et al. 2015; Xue et al., 2014). However, miR-155 has also been indicated to be down-regulated in various hematological malignancies: Burkitt' s lymphoma (Kluiver et al., 2006), CML (Rokah et al., 2012) and AML with inv(16) (Jongen-Lavrencic et al., 2008), indicating the complexity of miR-155 regulation in tumorigenesis. In consistent with Palma's study (Palma et al., 2014), we showed that miR-155 has a pro-differentiation and antiproliferation role in FLT3-WT AML cells, in contrast to its oncogenic function reported in B cell lymphoma (Eis et al., 2005) and FLT3-ITD mutation AML (Gerloff et al., 2015; Xue et al., 2014). Therefore, these evidences suggested that the gene regulation by miR-155 is complex (Palma et al., 2014), as it targets both tumor suppressor gene or oncogene, and its function in a specific malignancy is likely subject to disease context and cell type.

\section{ACKNOWLEDGMENTS}

This work was supported by grants from the National Natural Science Foundation of China (81260269) and Natural Science Foundation of Inner Mongolia People's Hospital (201567). 


\section{REFERENCES}

Alaiyan, B., llyayev, N., Stojadinovic, A., Izadjoo, M., Roistacher, M., Pavlov, V., Tzivin, V., Halle, D., Pan, H., Trink, B., et al. (2013). Differential expression of colon cancer associated transcript1 (CCAT1) along the colonic adenoma-carcinoma sequence. BMC Cancer 13, 196.

Arancio, W., Pizzolanti, G., Genovese, S.I., Baiamonte, C., and Giordano, C. (2014). Competing endogenous RNA and interactome bioinformatic analyses on human telomerase. Rejuvenation Res. 17, 161-167.

Calin, G.A., Ferracin, M., Cimmino, A., Di Leva, G., Shimizu, M., Wojcik, S.E., lorio, M.V., Visone, R., Sever, N.I., Fabbri, M., et al. (2005). A MicroRNA signature associated with prognosis and progression in chronic lymphocytic leukemia. N Engl J. Med. 353,1793-1801.

Deng, L., Yang, S.B., Xu, F.F., and Zhang, J.H. (2015). Long noncoding RNA CCAT1 promotes hepatocellular carcinoma progression by functioning as let-7 sponge. J. Exper. Clin. Cancer Res. 34, 18.

Eis, P.S., Tam, W., Sun, L., Chadburn, A., Li, Z., Gomez, M.F., , E., Dahlberg, J.E. (2005). Accumulation of miR-155 and BIC RNA in human B cell lymphomas. Proc. Natl. Acad. Sci. USA 102, 36273632.

Fernando, T.R., Rodriguez-Malave, N.I., Waters, E.V., Yan, W., Casero, D., Basso, G., Pigazzi, M., and Rao, D.S. (2015). LncRNA expression discriminates karyotype and predicts survival in BLymphoblastic Leukemia. Mol. Cancer Res. 13, 839-851.

Gerloff, D., Grundler, R., Wurm, A.A., Bräuer-Hartmann, D., Katzerke, C., Hartmann, J.U., Madan, V., Müller-Tidow, C., Duyster, J., Tenen, D.G. et al. (2015). NF-kB/STAT5/miR-155 network targets PU. 1 in FLT3-ITD-driven acute myeloid leukemia. Leukemia 29, 535-547.

Hughes, J.M., Legnini, I., Salvatori, B., Masciarelli, S., Marchioni, M., Fazi, F., Morlando, M., Bozzoni, I., and Fatica, A. (2015). C/ EBPalpha-p30 protein induces expression of the oncogenic long non-coding RNA UCA1 in acute myeloid leukemia. Oncotarget 6, 18534-18544.

Ichikawa, M., Asai, T., Saito, T., Seo, S., Yamazaki, I., Yamagata, T., Mitani, K., Chiba, S., Ogawa, S., Kurokawa, M., et al. (2004). AML1 is required for megakaryocytic maturation and lymphocytic differentiation, but not for maintenance of hematopoietic stem cells in adult hematopoiesis. Nat. Med. 10, 299-304.

Jeziskova, I., Musilova, M., Culen, M., Foltankova, V., Dvorakova, D., Mayer, J., and Racil, Z. (2015). Distribution of mutations in DNMT3A gene and the suitability of mutations in R882 codon for MRD monitoring in patients with AML. Int. J. Hematol. 102, 553557.

Johnsson, P., Ackley, A., Vidarsdottir, L., Lui, W.O., Corcoran, M., Grander, D., and Morris, K.V. (2013). A pseudogene longnoncoding-RNA network regulates PTEN transcription and translation in human cells. Nat. Struct. Mol. Biol. 20, 440-446.

Jongen-Lavrencic, M., Sun, S.M., Dijkstra, M.K., Valk, P.J.M., Löwenberg, B. (2008). MicroRNA expression profiling in relation to the genetic heterogeneity of acute myeloid leukemia. Blood 111, 5078-5085.

Kallen, A.N., Zhou, X.B., Xu, J., Qiao, C., Ma, J., Yan, L., Lu, L., Liu, C., Yi, J.S., Zhang, H., et al. (2013). The imprinted H19 IncRNA antagonizes let-7 microRNAs. Mol. Cell 52, 101-112.

Khandelwal, A., Bacolla, A., Vasquez, K.M., and Jain, A. (2015). Long non-coding RNA: A new paradigm for lung cancer. Mol. Carcinog. 54, 1235-1251.

Kluiver, J., Poppema, S., De Jong, D., Blokzill, T., Harms, G., Jacobs, S., Kroesen, B.J., and van den Berg, A. (2005). BIC and miR-155 are highly expressed in Hodgkin, primary mediastinal and diffuse large B cell lymphomas. J. Pathol. 207, 243-249.

Kluiver, J., Haralambieva, E., De Jong, D., Blokzijl, T., Jacobs, S., Kroesen, B.J., Poppema, S., and van den Berg, A. (2006). Lack of $\mathrm{BIC}$ and microRNA miR-155 expression in primary cases of Burkitt lymphoma. Genes Chromosomes Cancer 45, 147-153.

Kumar, M.S., Armenteros-Monterroso, E., East, P., Chakravorty, P Matthews, N., Winslow, M.M., and Downward, J. (2014). HMGA2 functions as a competing endogenous RNA to promote lung cancer progression. Nature 505, 212-217.

Mizrahi, I., Mazeh, H., Grinbaum, R., Beglaibter, N., Wilschanski, M., Pavlov, V., Adileh, M., Stojadinovic, A., Avital, I., Gure, A.O., et al.
(2015). Colon cancer associated transcript-1 (CCAT1) expression in adenocarcinoma of the stomach. J. Cancer 6, 105-110.

Palma, C.A., Al Sheikha, D., Lim, T.K., Bryant, A., Vu, T.T., Jayaswal, V., and Ma, D.D. (2014). MicroRNA-155 as an inducer of apoptosis and cell differentiation in acute myeloid leykaemia. Mol. Cancer. 13, 79

Peng, W., Si, S., Zhang, Q., Li, C., Zhao, F., Wang, F., Yu, J., and Ma R. (2015). Long non-coding RNA MEG3 functions as a competing endogenous RNA to regulate gastric cancer pro- gression. J. Exp. Clin. Cancer Res. 34, 79.

Poliseno, L., Salmena, L., Zhang, J., Cavver, B., Haveman, W.J., and Pandolfi, P.P. (2010). A coding-independent function of gene and pseudogene mRNAs regulates tumour biology. Nature 465, 10331038.

Rokah, O.H., Granot, G., Ovcharenko, A., Modai, S., Pasmanik-Chor, M., Toren, A., Shomron, N., and Shpilberg, O. (2012). Downregulation of miR-31, miR-155, and miR-564 in chronic myeloid leukemia cells. PLoS One 7, e35501.

Saadatpour, A., Guo, G., Orkin, S.H., and Yuan, G.C. (2014). Characterizing heterogeneity in leukemic cells using single-cell gene expression analysis. Genome Biol. 15, 525.

Sakurai, M., Kunimoto, H., Watanabe, N., Fukuchi, Y., Yuasa, S., Yamazaki, S., Nishimura, T., Sadahira, K., Fukuda, K., Okano, H., et al. (2014). Impaired hematopoietic differentiation of RUNX1mutated induced pluripotent stem cells derived from FPD/AML patients. Leukemia 28, 2344-2354.

Sen, R., Ghosal, S., Das, S., Balti, S., and Chakrabarti, J. (2014). Competing endogenous RNA: the key to posttranscriptional regulation. ScientificWorldJournal 2014, 896206.

Song, X., Cao, G., Jing, L., Lin, S., Wang, X., Zhang, J., Wang, M., Liu, W., and LV, C. (2014). Analysing the relationship between IncRNA and protein-coding gene and the role of IncRNA as ceRNA in pulmonary fibrosis. J. Cell. Mol. Med. 18, 991-1003.

Su, R., Lin, H.S., Zhang, X.H., Yin, X.L., Ning, H.M., Liu, B., Zhai, P.F., Gong, J.N., Shen, C., Song, L., et al. (2015). MiR-181 family: regulators of myeloid differentiation and acute myeloid leukemia as well as potential therapeutic targets. Oncogene 34, 3226-3239.

Sun, S., Sun, P., Wang, C., and Sun, T. (2014). Downregulation of microRNA-155 accelerates cell growth and invasion by targeting cmyc in human gastric carcinoma cells. Oncol. Rep. 32, 951-956.

Wang, J., Liu, X., Wu, H., Ni, P., Gu, Z., Qiao, Y., Chen, N., Sun, F., and Fan, Q. (2010). CREB up-regulates long non-coding RNA, HULC expression through interaction with microRNA-372 in liver cancer. Nucleic Acids Res. 38, 5366-5383.

Wang, Y., Wu, P., Lin, R., Rong, L., Xue, Y., and Fang, Y. (2015). LnCRNA NALT interaction with NOTCH1 promoted cell proliferation in pediatric T cell acute lymphoblastic leukemia. Sci. Rep. 5, 13749.

Xue, H., Hua, L.M., Guo, M., and Luo, J.M. (2014). SHIP1 is targeted by miR-155 in acute myeloid leukemia. Oncol. Rep. 32, 2253-2259.

Yang, F., Xue, X., Bi, J., Zheng, L., Zhi, K., Gu, Y., and Fang, G. (2013). Long noncoding RNA CCAT1, which could be activated by c-Myc, promotes the progression of gastric carcinoma. J. Cancer Res. Clin. Oncol. 139, 437-445.

Ye, N., Wang, B., Quan, Z.F., Cao, S.J., Wen, X.T., Huang, Y., Huang, X.B., Wu, R., Ma, X.P., and Yan, Q.G. (2014). Functional roles of long non-coding RNA in human breast cancer. Asian Pac. J. Cancer Prev. 15, 5993-5997.

Yokoyama, Y., Wan, X., Shinohara, A., Takahashi, S., Takahashi, Y., Niwa, K., and Tamaya, T. (2000). Expression of PTEN and PTEN pseudogene in endometrial carcinoma. Int. J. Mol. Med. 6, 47-50.

Yu, G., Yao, W., Gumireddy, K., Li, A., Wang, J., Xiao, W., Chen, K., Xiao, H., Li, H., Tang, K., et al. (2014). Pseudogene PTENP1 functions as a competing endogenous RNA to suppress clear-cell renal cell carcinoma progression. Mol. Cancer Ther. 13, 3086-3097.

Yuan, J.H., Yang, F., Wang, F., Ma, J.Z., Guo, Y.J., Tao, Q.F., Liu, F., Pan, W., Wang, T.T., Zhou, C.C., et al. (2014). A long noncoding RNA activated by TGF-beta promotes the invasion-metastasis cascade in hepatocellular carcinoma. Cancer Cell 25, 666-681.

Zhang, H., Chen, Z., Wang, X., Huang, Z., He, Z., and Chen, Y. (2013). Long non-coding RNA: a new player in cancer. J. Hematol. Oncol. 6, 37

Zhu, H., Zhou, X., Chang, H., Li, H., Liu, F., Ma, C., and Lu, J. (2015). CCAT1 promotes hepatocellular carcinoma cell proliferation and invasion. Int. J. Clin. Exper. Pathol. 8, 5427-5434. 